\title{
ESTIMATION AND CONTROL OF A DOUBLE-INVERTED PENDULUM
}

\author{
Bowen $\mathrm{Xu}^{*}$, You Lyu, and S. A. Gadsden \\ College of Engineering and Physical Sciences, \\ University of Guelph, Ontario, Canada \\ *bxu04@uoguelph.ca
}

\begin{abstract}
This paper studies estimation and control methods applied on a double-inverted pendulum. The most popular estimation strategy, referred to as the Kalman filter (KF), was programmed and implemented on a double-inverted pendulum built by Quanser (Markham, Ontario). A linear quadratic regulator (LQR) was used to control the actuator joints of the double-inverted pendulum to keep it vertically balanced under different conditions. The application of the KF improved the tracking performance. The results of the paper are discussed, and future work is considered.
\end{abstract}

Keywords-double inverted pendulum; control system; Kalman filter; estimation.

\section{INTRODUCTION}

The double-inverted pendulum is one of the most popular mechatronic research systems used to study control and estimation strategies. The system itself is relatively easy to build, and is naturally unstable which makes it quite popular to study. The complex dynamics of a double-inverted pendulum offers a system suitable for the development and examination of new control and estimation methodologies. The findings can be applied to problems in current research, such as holding the balance of a robot or rotor-based aircraft [1]. In order to improve the control performance of the system, estimation strategies can be used to provide better knowledge of the states [1].

State and parameter estimation theory is an important field in mechanical and electrical engineering. The strategies are used to predict, estimate, or smooth out important system state and parameters [2,3]. The Kalman filter (KF) is the most popular estimation strategy, and yields a statistically optimal solution to the linear estimation problem [4]. The goal of the KF is to minimize the state error covariance, which is a measure of the estimation accuracy and is defined as the expectation of the state error squared [5]. A popular nonlinear form of the Kalman filter is the unscented Kalman filter (UKF). It utilizes statistics to approximate the nonlinear probability density function (PDF) [6]. These filters can be applied on mechanical or electrical systems to improve the overall control tracking performance [4].

The brief paper is organized as follows. In Section II, the linear-quadratic regulator (LQR) controller, the standard $\mathrm{KF}$, and the MATLAB simulation is summarized. The application results are provided and discussed in Section III. Conclusions and future work are considered in the final section of the paper.

\section{Methodology}

\section{A. Model and Linear-quadratic regulator (LQR) control}

The double-inverted pendulum has a complex dynamic system [7]. In order to improve the control performance (e.g., balancing the system dynamics), a linear-quadratic regulator control strategy is applied in this paper. Quanser (Markham, Ontario) built the double-inverted pendulum system used in this paper [7]. The results of this paper were built upon this platform and the provided software. The first step of applying the LQR control strategy is to obtain the state-space model of the system. The motion equations may be obtained by using the following Euler-Lagrange equation, as follows [7]:

$$
Q_{i}=\frac{\partial L}{\partial t \partial \partial_{\dot{q} 1}}-\frac{\partial L}{\partial q_{i}}
$$

The generalized coordinate $q_{i}$ may be found as follows [7]:

$$
\begin{aligned}
& q(t)^{T}=\left[\begin{array}{lll}
x_{c}(t) & \alpha(t) & \theta(t)
\end{array}\right] \\
& \dot{q}(t)^{T}=\left[\begin{array}{lll}
\frac{\partial x_{c}(t)}{\partial t} & \frac{\partial \alpha(t)}{\partial t} & \frac{\partial \theta(t)}{\partial t}
\end{array}\right]
\end{aligned}
$$

where the above parameters are defined as follows [7]:

$$
\begin{aligned}
& Q_{1}=\frac{\partial^{2} L}{\partial t \partial \dot{x}_{c}}-\frac{\partial L}{\partial x_{c}} \\
& Q_{2}=\frac{\partial^{2} L}{\partial t \partial \dot{\alpha}}-\frac{\partial L}{\partial \alpha} \\
& Q_{3}=\frac{\partial^{2} L}{\partial t \partial \dot{\theta}}-\frac{\partial L}{\partial \theta}
\end{aligned}
$$

The Lagrange (or energy) equation for the double-inverted pendulum system is defined by:

$$
L=T-V
$$

In equation (7), $T$ refers to the kinetic energy of the doubleinverted pendulum system, $V$ is the total potential energy of the double-inverted pendulum system. According to equations (4) through (7), the generalized forces of the system may be found as follows [7]:

$$
\begin{gathered}
Q_{1}=F_{c}-B_{e q} \dot{x_{c}} \\
Q_{2}=-B_{p 1} \dot{\alpha} \\
Q_{3}=-B_{p 2} \dot{\theta}
\end{gathered}
$$


After obtaining the nonlinear motion equations, a Jacobian matrix is calculated and the linear state-space model may be found as follow:

$$
\begin{aligned}
& \dot{x}=A x+B u \\
& y=C x+D u
\end{aligned}
$$

where $x$ refers to states, $u$ is the input from the motor, $A$ is the system matrix, $B$ is the input matrix, $C$ is the measurement matrix, and $D$ is the feedforward matrix. The state and output equations are respectively as follows:

$$
\begin{gathered}
x^{T}=\left[\begin{array}{llllll}
x_{c} & \alpha & \beta & \dot{x}_{c} & \dot{\alpha} & \dot{\theta}
\end{array}\right] \\
y^{T}=\left[\begin{array}{lllll}
x_{1} & & x_{2} & & x_{3}
\end{array}\right]
\end{gathered}
$$

After obtaining the linear state-space matrices, the LQR controller can be implemented. The LQR control strategy can provide full feedback control of the system [4]. The LQR equation is defined by [7]:

$$
J=\int_{0}^{\infty} x(t)^{\prime} Q x(t)+u(t)^{\prime} R u(t) d t
$$

In equation (15), $Q$ and $R$ are system and measurement noise covariances, respectively:

$$
Q=\left[\begin{array}{ccccccc}
10 & 0 & 0 & 0 & 0 & 0 & 0 \\
0 & 50 & 0 & 0 & 0 & 0 & 0 \\
0 & 0 & 50 & 0 & 0 & 0 & 0 \\
0 & 0 & 0 & 0 & 0 & 0 & 0 \\
0 & 0 & 0 & 0 & 0.9 & 0 & 0 \\
0 & 0 & 0 & 0 & 0 & 0.9 & 0 \\
0 & 0 & 0 & 0 & 0 & 0 & 1
\end{array}\right]
$$

In order to reduce regulation error of the linear cart, an integral term is added:

$$
\begin{aligned}
& \eta=\left[\begin{array}{ll}
A & 0 \\
1 & 0
\end{array}\right] \eta+\left[\begin{array}{c}
B \\
0
\end{array}\right] u \\
& {\left[\begin{array}{lllllll}
x_{c}-x_{c, d} & \alpha & \theta & \dot{x}_{c} & \dot{\alpha} & \dot{\theta} & \int\left(x_{c}-x_{c, d}\right) d t
\end{array}\right]}
\end{aligned}
$$

According to the control law, the equation of control feedback of this double-inverted pendulum may be found as follows:

$$
u=-K(\eta)
$$

There are three important model characterizations for control of the double-inverted pendulum: observability, controllability, and stability. Observability of a system states can be observed from the system output [8]. In this paper, the system is observable but not completely observable because the measurement matrix is a non-square matrix. The dynamic system is said to be controllable which means for any desired final state, the system can be driven by the input [8]. In this paper, by calculating the rank of the controllability matrix, the controllability can be proven. The system is stable if every bounded input produces a bounded output (principle of BIBO stability). In this paper, the stability was calculated using Maple, because some poles are positive, the system is considered to be unstable [9].

\section{B. Kalman Filter and Matlab Simulink}

For the control of the double-inverted pendulum, the cart position, and both pendulum angles are measured. According to the measurements, a voltage is determined that is used by the control system to actuate the cart's motor. As a result, the cart balances the double inverted pendulum and keeps the upright, vertical position.

For the software, the programs Matlab and Simulink were used. For this system, the controller could only be implemented in Simulink, whereas the programming environment of Matlab offers more possibilities. The workstation provides specialized Simulink blocks to communicate with the pendulum control. In real-time, the measurements of the position and angles are processed to control the motor by setting the voltage (as per the Figure 1). Simulink was used as the interface with the workstation, and Matlab as the data processor and background algorithms. The goal of this setup was to go beyond the setup provided commercially by Quanser in [7], and create a setting that enables real-time signal processing and the capability to add features over the time without losing the overview.

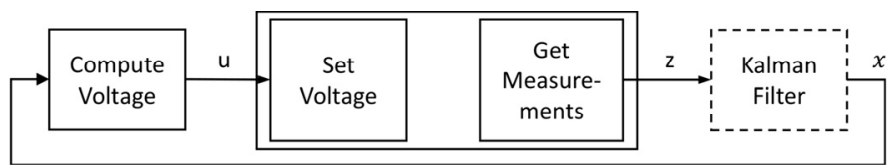

Figure 1. Control loop of the double-inverted pendulum.

\section{Implementation of the Kalman Filter}

For the Kalman filter, a linear, discrete-time, and timeinvariant system model is used [10]:

$$
\begin{gathered}
x_{k+1}=A x_{k}+B u_{k}+w_{k} \\
z_{k+1}=C x_{k+1}+v_{k+1}
\end{gathered}
$$

The Kalman filter is based on two stages: prediction and update. In each stage, the state vector and its covariance matrix are calculated. To simplify the notation, we write for the expected value of the a-priori density of the prediction as follows:

$$
E\left\{x_{k+1} \mid z_{k}\right\}=\dot{x}_{k+1}
$$

For the expected value of the a-posteriori density of the update as follows:

$$
E\left\{x_{k+1} \mid z_{k}\right\}=\hat{x}_{k+1}
$$

The estimation criterion is the minimization of the square norm if the estimation error:

$$
E\left\{\left|\hat{x}(n)-x(n)^{2}\right| z(n)\right\} \rightarrow \min
$$

With the linearized matrices of the dynamic coefficient matrix $A$, the coupling matrix $B$, and the measurement matrix $C$, we can use the extended Kalman filter (EKF) to decrease our measurement deviation. For the computation, the equations are shown below [11]:

Prediction stage:

$$
\begin{gathered}
\hat{x}_{k+1 \mid k}=A \hat{x}_{k \mid k}+B u_{k} \\
P_{k+1 \mid k}=A P_{k \mid k} A^{T}+Q_{k}
\end{gathered}
$$


Update stage:

$$
\begin{gathered}
K_{k+1}=P_{k+1 \mid k} C^{T}\left(C P_{k+1 \mid k} C^{T}+R_{k+1}\right)^{-1} \\
\hat{x}_{k+1 \mid k+1}=\hat{x}_{k+1 \mid k}+K_{k+1}\left[z_{k+1 \mid k}-C \hat{x}_{k+1 \mid k}\right] \\
P_{k+1 \mid k+1}=\left(I-K_{k+1} C\right) P_{k+1 \mid k}
\end{gathered}
$$

The unscented Kalman filter (UKF) belongs to the group of sigma point Kalman filter, which uses a weighted statistical linear regression strategy that approximates the nonlinear model statistically [6].

\section{Implementation of the Developed Interface}

The Kalman filters are implemented in a Matlab block in Simulink. The advantage is its simplicity. The block has to be created, the function to be written and the connection of inputs and outputs to be made. The drawbacks are the computation time that might be slower in comparison with the usage of Simulinkonly state space blocks. Nevertheless, the Matlab function block can be used. The value of the gain 'FilterChoice' can be modified using the user interface. With the if-operation and the modification of the choice input, different code sections can be executed, performing different KF options. The voltage input for the KF is provided by a 'Data Store Read' block. If we would connect the input $u$ with the output of the voltage determination step, we would create an algebraic loop. Due to the discrete state space model, the computation has to be executed in a finite time step. By calling itself, an infinite circle is created making a discrete state space computation impossible. Due to this, instead of initiating the execution of the KF function by the arrival of the voltage input, we write the input $u$ in a 'Data Store Write' block and the value can be read out if the measurements initiate the execution of the block.

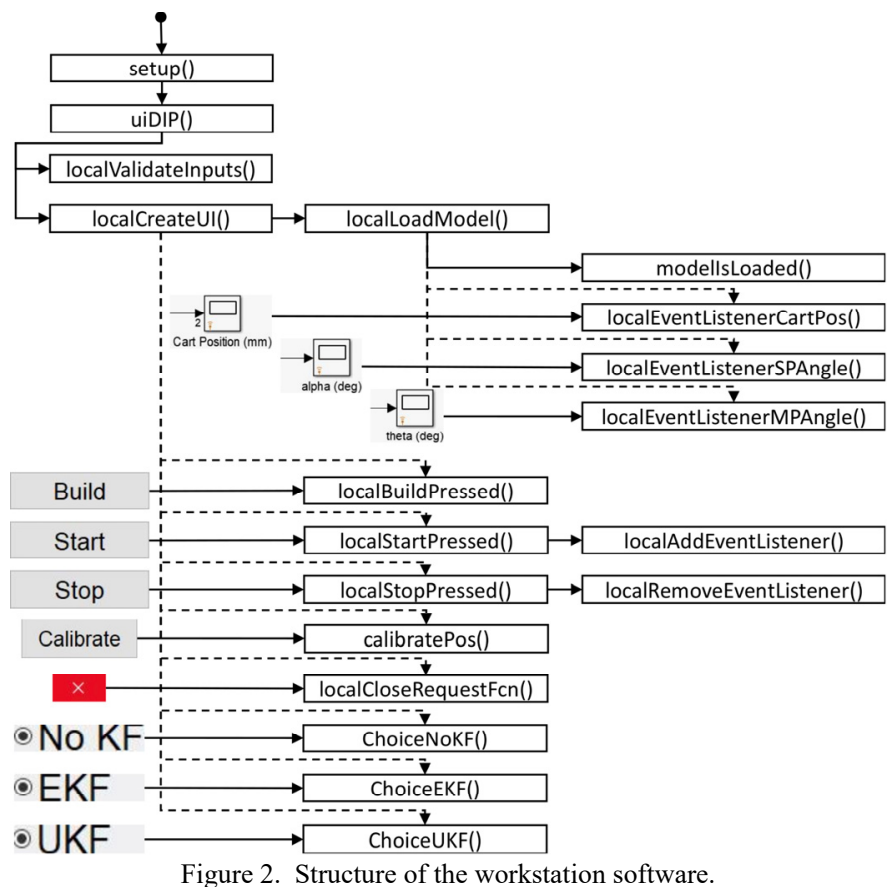

Matlab can modify the Simulink model and the user interface during the simulation by changing the parameters in Matlab's data structure. In the opposite way, Matlab obtains the data from
Simulink over event-listeners and from the user interface over callbacks. In order to accomplish this, the event listeners and callback functions have to be implemented first. The function 'localCreateUI' creates the figure of the user interface with all components and adds callback functions that are executed, each time the buttons are pressed. Additionally, this function also executes the function 'localLoadModel', which implements the event-listeners for the measurements of the cart position and the angles of the short and medium pendulum. Each time, a value arrives at one of the event-listener blocks, the event-listener function in Matlab is executed. By this approach, a bidirectional connection between the Simulink model and the user interface over the model is possible.

\section{SIMULATION RESULTS}

\section{A. Software Simulation}

The software only simulation is based on Matlab and Simulink. It provides software-based system running simulation for this double inverted pendulum system. All of the important parameters of the system such as output voltage, cart position, and pendulum angles are visible by real time graphs. Software only simulations can be roughly divided into ideal and non-ideal cases. Ideal types do not have system and measurement noises included in the simulation. While the non-ideal cases take all these factors in count and the results are closer to real system. In this paper, we test system performance for all these situations.

The system is built in Simulink and all components are represented by functional blocks. There are two key parts in the system design, namely LQR balance controller and the pendulum system. The pendulum system itself can be simulated in several ways. Regardless, using any method to simulate the pendulum system, there is only one input (motor voltage) for this system. The three output states are cart position and angles of the two pendulums.

\section{1) Ideal Case}

This specific simulation employs an ideal system model, which contains no noise interference. The results should be ideal inputs and output which will be considerably different from the hardware-connected case. However, this insight-view provides a convenient way to become familiar with the system and can benefit the configuration and optimization of the physical system. Simulink is the platform to perform this work. All of the parameters will be monitored in Simulink scopes. The Matlab file provides all the matrices and parameters of the system. The operation of the Simulink model is based on content loaded into Matlab's workspace. The pendulum system itself is represented by the state space model. Data flow for this target mainly contains the input, which is the voltage applied to the cart motor. The output are the three states of the system, cart position, angle of the first pendulum and the angle of the second pendulum, respectively. LQR balance control is the selected controller in this paper; however, the popular PID controller could also be used. The LQR part sends the calculated voltage values to control the motor motions. The simulation performed is set to contain two position values: the cart is supposed to stay at one place for ten seconds, then move to the second position and stay there for the next ten seconds, before returning to the original position. The simulation results are shown next. 


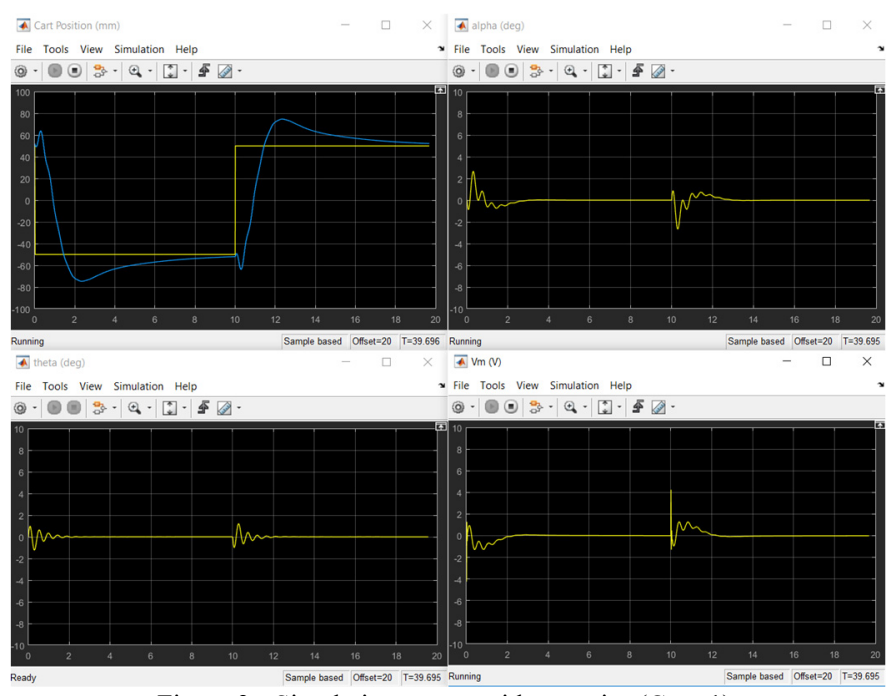

Figure 3. Simulation output without noise (Case 1).

In Figure 3(top-left), the yellow curve is the position setting and the blue curve is the cart output. The simulated cart moves in a rather smooth manner to reach the settling points. Compared with the yellow curve, the blue curve shows reasonable overshoot and low steady state error. The joint angles and voltage show small fluctuations, only occurs when the cart moves from one location to another.

\section{2) Noisy Measurement Case}

In this case, noisy measurements are considered and added to the simulation. In order to improve the control results, the Kalman filter was added to estimate the states. The noisy signal with filtered results are shown in Figure 4.

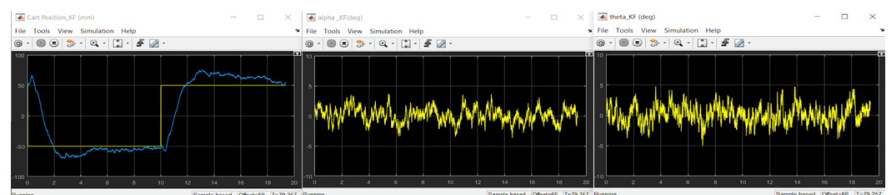

Figure 4. Simulation output with the KF (Case 2).

The results contain small fluctuations during the stabilization process of the cart (after quick position movements). The simulation shows that the steady-state error stays approximately at the same level compared with the ideal case. In contrast, the angles were more unstable. However, unlike the ideal case, the fluctuations never settle.

\section{3) System with Process and Measurement Noise}

In this case, the simulation contains both system and measurement noise. The cart position results without the addition of the KF shows significantly more fluctuations compared to the ideal case. The angles reveal the same trend as the cart position. In contrast, the addition of the KF shows more fluctuations which means the cart requires additional movements to keep the system balanced.

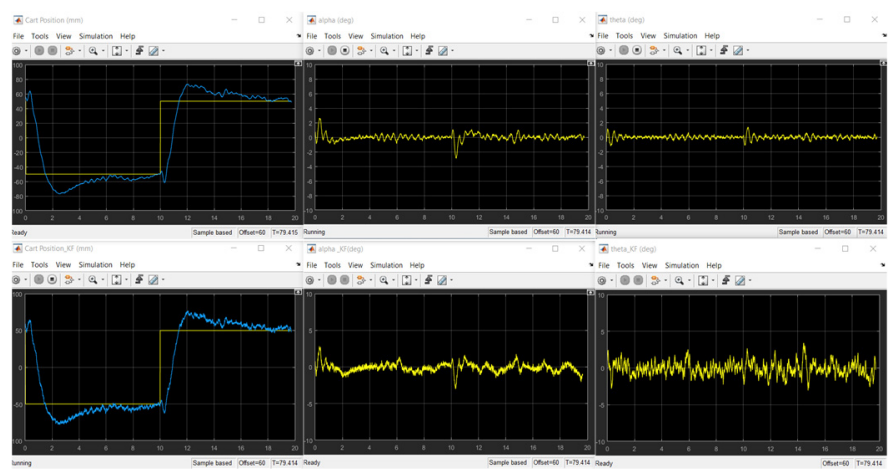

Figure 5. Simulation output with the KF (Case 3).

\section{B. Experimental System and Software Simulation}

This section focuses on the simulation with the pendulum. The motion and status of the real system is considerably different from the simulated type. The goal is to achieve and demonstrate the successful control of the double-inverted pendulum system.

\section{1) Normal Case}

In case, consider the system noise covariance matrix as per equation (16) and the measurement uncertainty covariance matrix as per equation (17). The normal case results are shown in the following figure.

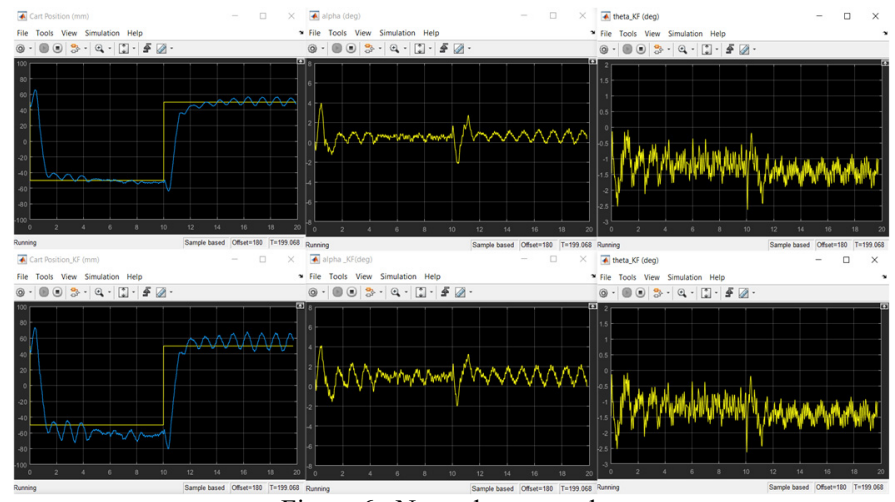

Figure 6. Normal case results.

The movement of the cart is fast and steady. The cart moves forward and backward in small scales to keep the balance of both pendulums. The angle values fluctuate as the cart moves to keep the whole system balanced. The addition of the KF improved the overall steady-state error of the system. Note also that the two angle states were quite similar in these two cases.

\section{2) Noisy Case}

The noisy system cases were simulated by increasing the noise covariance. The results are shown as per Figure 7. The system noise covariance matrix was changed as follows:

$$
Q=\left[\begin{array}{ccccccc}
30 & 0 & 0 & 0 & 0 & 0 & 0 \\
0 & 350 & 0 & 0 & 0 & 0 & 0 \\
0 & 0 & 100 & 0 & 0 & 0 & 0 \\
0 & 0 & 0 & 0 & 0 & 0 & 0 \\
0 & 0 & 0 & 0 & 0.5 & 0 & 0 \\
0 & 0 & 0 & 0 & 0 & 0.5 & 0 \\
0 & 0 & 0 & 0 & 0 & 0 & 1
\end{array}\right]
$$



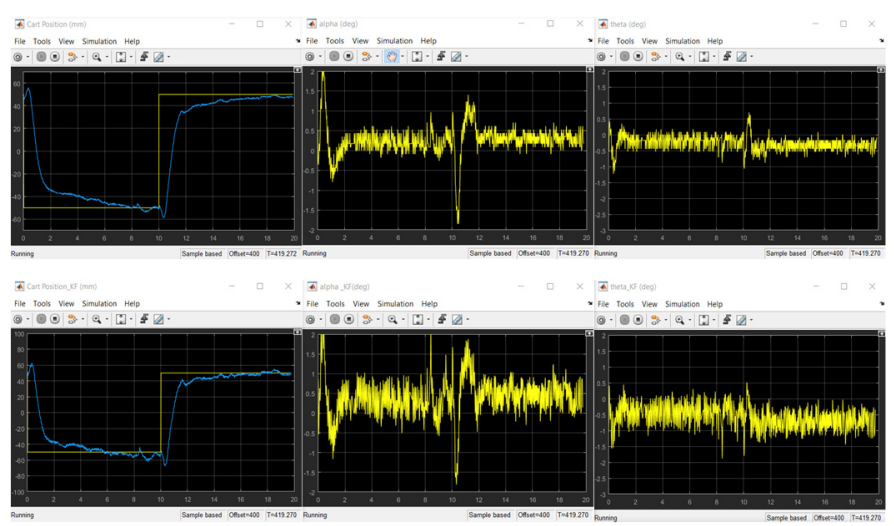

Figure 7. Noisy case results.

The cart moves quickly but in small movements in this case. The addition of the KF to the system improves the system performance, and the required settling time was decreased. It is important to note that both the LQR and the LQR with the KF methods were able to balance the double-inverted pendulum system. However, the LQR with KF method had improved response and faster settling time.

\section{CONCLUSIONS AND FUTURE WORK}

In this brief paper, the results of applying a controller and estimation strategy on a double-inverted pendulum were summarized and discussed. With well-defined parameters and a well-tuned KF, the system is able to overcome instabilities and external forces. The results demonstrate that the KF results are closer to the true values, which means that the LQR control strategy with the KF provides a better performance for the double-inverted pendulum system. Future work will look at implementing other control strategies, such as an adaptive PID, and comparing the results.

\section{REFERENCES}

[1] H. Wang and S. Jiang, "LQR Control of Single Inverted Pendulum Based on Square Root Filter," Advanced Materials Research, Vols. 433-440, pp. 6999-7003, 2012.

[2] B. D. O. Anderson and J. B. Moore, Optimal Filtering, Englewood Cliffs, NJ: Prentice-Hall, 1979.

[3] Y. Bar-Shalom, X. Rong Li and T. Kirubarajan, Estimation with Applications to Tracking and Navigation, New York: John Wiley and Sons, Inc., 2001.

[4] S. A. Gadsden, "Smooth Variable Structure Filtering: Theory and Applications," Ph.D. Thesis, McMaster University, Hamilton, Ontario, 2011.

[5] M. S. Grewal and A. P. Andrews, Kalman Filtering: Theory and Practice Using MATLAB, 3 ed., New York: John Wiley and Sons, Inc., 2008.

[6] S. A. Gadsden and A. S. Lee, "Advances of the Smooth Variable Structure Filter: Square-Root and Two-Pass Formulations," Journal of Applied Remote Sensing, vol. 11, no. 1, pp. 1-19, 2017.

[7] Quanser, "Linear Motion Servo Plants: IP012 DBLP Laboratory Manual," Markham, Ontario, 2017.

[8] B. Moore, "Principal Component Analysis in Linear Systems: Controllability, Observability, and Model Reduction," IEEE Transactions on Automatic Control, vol. 26, no. 1, pp. 17-32, 1981.

[9] N. Nise, Control Systems Engineering, 4 ed., New York: John Wiley and Sons, Inc., 2004.

[10] S. A. Gadsden and S. R. Habibi, "A New Form of the Smooth Variable Structure Filter with a Covariance Derivation," in IEEE Conference on Decision and Control, Atlanta, Georgia, 2010.

[11] S. A. Gadsden and S. R. Habibi, "A New Robust Filtering Strategy for Linear Systems," ASME Journal of Dynamic Systems, Measurement, and Control, vol. 135, no. 1, p. 014503, 2012. 